
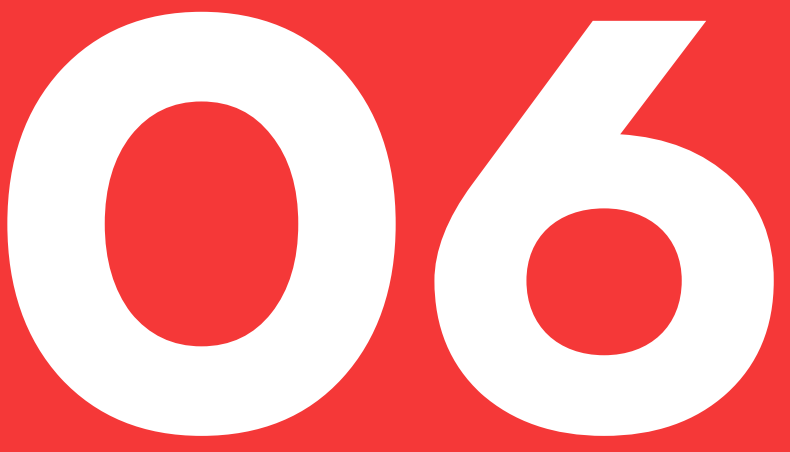

\title{
La sostenibilidad del concepto de Desarrollo Sostenible. ¿Cómo hacerlo operativo?
}

The sustainability of the concept of Sustainable Development.

How to make it operational?

Fecha de recepción: 26/008/2019

Fecha de aprobación: 18/07/2020 


\section{Resumen}

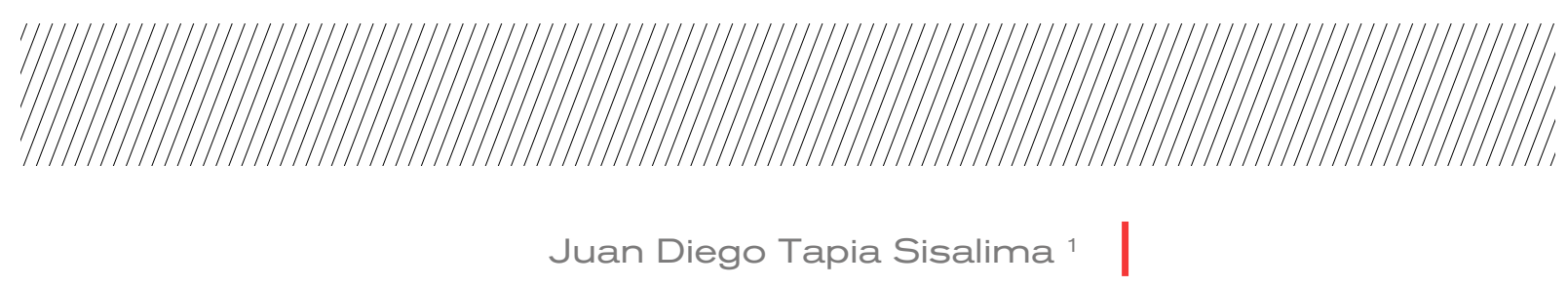

Los desafíos del Antropoceno generan presiones por la planificación, fomento y aplicación de innovadores cambios económicos, éticos, políticos y sociales. La propuesta del Desarrollo Sostenible, a pesar

Investigador independiente de insistir en un crecimiento económico ilimitado con fundamento en la teoría capitalista, y, de su enfoque antropocéntrico, a través de la Agenda 2030 y sus Objetivos de Desarrollo Sostenible (ODS) se ha establecido como el punto de referencia para el desarrollo global futuro. No obstante, las críticas al concepto y su operatividad son varias. El presente ensayo ofrece una reflexión crítica de este concepto y de su propia sostenibilidad y, a partir de ella, con relación a los ODS y su interconexión con la gestión integrada de recursos hídricos (GIRH), se discute de manera descriptiva la forma de hacerlo operativo a través del análisis con indicadores de gestión. Enfoques holísticos y complejos son comunes entre Desarrollo Sostenible y GIRH, por lo que sus dinámicas y abordajes hacia la operatividad en el ámbito de la sostenibilidad, también podrían serlo.

Palabras Clave

Antropoceno, Desarrollo Sostenible, Economía Política, Naturaleza,

Recursos Hídricos. 


\section{Abstract}

- he challenges of the Anthropocene generate pressures for the planning, promotion and application of pioneering economic, ethical, political and social changes. The

Sustainable Development proposal, despite persist on unlimited economic growth based on capitalist theory, and, of its anthropocentric viewpoint, through the 2030 Agenda and its Sustainable Development Goals (SDGs) has been established as the point of reference for future global development. Nevertheless, there are several criticisms of the concept and its operability. This essay offers a critical reflection of this concept and of its own sustainability, and based on it, in relation to the SDGs and their interconnection with the integrated management of water resources (IWRM), the way to make it operational is discussed in a descriptive way through the analysis with management indicators. Holistic and complex outlooks are common between Sustainable Development and IWRM, so their dynamics and approaches to operability in the field of sustainability could also be.

\section{Keywords:}

Anthropocene, Sustainable Development, Political Economy, Nature, Water Resources 


\section{La sostenibilidad del concepto de Desarrollo Sostenible. ¿Cómo hacerlo operativo?}

$\mathrm{L}$

os paradigmas y enfoques antropocéntricos que históricamente han orientado las generalizadas percepciones de la humanidad respecto a la Naturaleza y a su lugar en el mundo, han fijado las profundas raíces del decadente carácter del modelo de producción y consumo de la actualidad (Alburquerque, 2010, 2013). Por un lado, la Naturaleza y su estado de crisis (evidenciado por dos problemas ambientales globales principales: el cambio climático y la alarmante tasa de pérdida de diversidad biológica); y, por otro, la creciente insatisfacción social y económica de millones de personas que no pueden cubrir sus necesidades básicas de bienestar personal y familiar; han afirmado grandes dudas y alertas respecto a estos modos de entender la vida, generando presiones por la planificación, fomento y aplicación de innovadores cambios económicos, éticos, políticos y sociales que nos involucran a todos (Gudynas, 2011; Jeanrenaud \& Jeanrenaud, 2018; Molina, Sarukhán, \& Carabias, 2017; Sachs, 2015). En respuesta a ello, se han propuesto alternativas frente a los retos que implica el Antropoceno.

La celebración de la Conferencia de las Naciones Unidas sobre el Medio Humano en Estocolmo en el año 1972, conjuntamente con la publicación simultánea del informe Los límites del crecimiento, son un hito histórico en materia de política internacional al plantear, por primera vez, en la agenda global, el problema complejo: economía - desarrollo - ambiente, reconociendo a este último como una cuestión de interés constante para el mundo (Díaz \& Escárcega, 2009). El Desarrollo Sostenible es un concepto que comenzó a tomar forma en tal época, no obstante, casi medio siglo después, el mundo está aún lejos de alcanzarlo (Sachs, 2015). A partir de una revisión bibliográfica rigurosa, desde el enfoque de las ciencias socioambientales, el presente ensayo ofrece una reflexión crítica del concepto de desarrollo sostenible y de su propia sostenibilidad, para discutir después, de manera descriptiva, la propuesta de los Objetivos de Desarrollo Sostenible de la Agenda 2030 y la manera de hacer operativo al concepto, tomando como caso de análisis su interconexión con la gestión integrada de los recursos hídricos. 


\section{Reflexión crítica del concepto}

Es preciso iniciar con un poco de historia relativa al concepto de Desarrollo Sostenible. En este aspecto, la concientización acerca de un evidente y acelerado deterioro medioambiental y de recursos naturales a nivel mundial, con negativas consecuencias para el desarrollo económico y social, motivó a que, en el año 1983, la Organización de las Naciones Unidas (ONU) creara la Comisión Mundial del Medio Ambiente y del Desarrollo.

Entre los objetivos de la Comisión estaban: i) proponer estrategias medioambientales a largo plazo; ii) recomendar acciones para la cooperación internacional que condujeran al establecimiento de objetivos globales comunes; iii) examinar medios para el tratamiento eficaz de problemas medioambientales; $y$, iv) elaborar un plan de acción para resolver los problemas vinculados a la protección y el mejoramiento del medio ambiente. De acuerdo a la resolución de su creación, la Comisión tenía que presentar un informe que debía incluir explícitamente "proyectos de estrategias para lograr un desarrollo duradero" (Naciones Unidas, 1982, 1987).

Empero, se debe recalcar que uno de las principales motivantes para la creación de la Comisión fue el aspecto económico, incluyendo a la Naturaleza -y su deterioro- únicamente como otro más de los factores de riesgo para su crecimiento, recayendo dentro de un enfoque desarrollista y de perspectiva utilitarista clásico. Así, tras mantener encuentros participativos a nivel global, la Comisión liderada por la ex primera ministra noruega Gro Harlem Brundtland, presentó en el año 1987 al principal órgano de la ONU, su Asamblea General, el Informe titulado Nuestro futuro común. Coloquialmente llamado Informe Brundtland, el documento es especialmente conocido por utilizar, por primera, vez el término 'Desarrollo Sostenible', al indicar que:

Está en manos de la humanidad hacer que el desarrollo sea sostenible, duradero, o sea, asegurar que satisfaga las necesidades del presente sin comprometer la capacidad de las futuras generaciones para satisfacer las propias. El concepto de desarrollo duradero implica límites - 
no límites absolutos, sino limitaciones que imponen a los recursos del medio ambiente el estado actual de la tecnología y de la organización social y la capacidad de la biósfera de absorber los efectos de las actividades humanas. Pero tanto la tecnología como la organización social pueden ser ordenadas y mejoradas de manera que abran el camino a una nueva era de crecimiento económico. (Naciones Unidas, 1987, p.23)

Conforme al Informe Brundtland, el Desarrollo Sostenible se fundamenta en tres pilares: desarrollo económico, desarrollo social y protección medioambiental, reconociendo las profundas interconexiones existentes entre estos sistemas (Naciones Unidas, 1987). En su dimensión económica, propone mantener y optimizar el desarrollo económico para, de esa manera, maximizar el bienestar humano considerando las limitaciones del capital natural, transformando los recursos económicos en sistemas rentables de trabajo mediante el logro de la autorrealización empresarial; en su dimensión social, busca la satisfacción equitativa y equilibrada de las necesidades intrageneracionales e intergeneracionales invitando al equilibrio de los objetivos y aspiraciones del consumidor; $y$, en su dimensión ambiental recalca que la Naturaleza no es una fuente inagotable de recursos y que el futuro del desarrollo depende de su conocimiento y manejo adecuado, velando por su protección y uso racional (Díaz \& Escárcega, 2009; Sachs, 2015).

En este contexto, se destaca que el concepto de Desarrollo Sostenible insiste en un crecimiento económico ilimitado, tal como la teoría económica capitalista (Méndez, 2005), con fundamento en una 'ordenación' de los sistemas tecnológicos y sociales y un 'manejo adecuado' de la Naturaleza, con una visión claramente antropocéntrica (Jeanrenaud \& Jeanrenaud, 2018). El concepto insta a perseguir la satisfacción de necesidades básicas de todos, con énfasis en brindar la oportunidad de colmar las aspiraciones a una vida mejor. Según el Informe Brundtland, esta satisfacción de necesidades exige no solo una nueva era de crecimiento económico para las naciones donde los pobres constituyen la mayoría, sino la garantía de que estos pobres dispongan de los recursos necesarios para sostener dicho crecimiento (Naciones Unidas, 1987). En este punto, su principal crítica -y la más frecuente- se evidencia en el hecho de que el concepto resulta paradójico, pues, persigue un crecimiento económico ilimitado, lo que no es ni ha sido, sostenible, ya que en más de medio siglo de imple- 
mentación alrededor del planeta, este modelo de desarrollo no ha logrado disminuir la brecha entre países ricos ni pobres, ni la cantidad de pobreza sino, más bien, ha resultado en un mayor número de países pertenecientes a un núcleo económico dominante (Carpio, 2019; Rodríguez \& Sánchez, 2019). Si bien, con base en la sostenibilidad se han generado beneficios específicos en materia de renta y oportunidades de empleo, graves externalidades negativas, ambientales y sociales, han planteado como uno de los problemas más complejos del Antropoceno, el cómo seguir generando riqueza sin afectar nuestro entorno (Salas, 2018). Así, la puesta en práctica de las loables propuestas del concepto, ha sido limitada por los intereses creados desde y hacia el poder económico-empresarial, sus esquemas prevalecientes y sus extensiones pragmáticas: el consumismo y el extractivismo, tergiversándolo hacia un discurso políticamente correcto únicamente (Carpio, 2019; Rodríguez \& Sánchez, 2019).

En relación a ello y respecto a su dimensión ambiental, el concepto propuesto se ha convertido en una crítica moderada e incompleta que ha resultado, por lo general, en planes de gobiernos nacionales que nunca han pasado de ser débiles estrategias impulsadas únicamente por las áreas ambientales, evidenciando una falta de política ambiental seria, coherente y articulada (Carpio, 2019). Si bien, el concepto de Desarrollo Sostenible se manifiesta en contra de la destrucción de ecosistemas naturales y de sus servicios como medio para el desarrollo con la justificación de metas productivas, energéticas o de servicios, también supone el concepto de límites del medio ambiente, conocidos como 'puntos quiebre ecosistémico' o 'tipping points'. Una economía basada en estas conceptualizaciones es sumamente compleja y poco resiliente, pues, su diseño se debería fundamentar en una base científica sólida en el aspecto referente a dotaciones y ritmos de consumo/agotamiento de materiales abióticos/bióticos y de energía en los ecosistemas de la Naturaleza (Bermejo, 2014). Es imperativo recalcar la enorme complejidad que implica el conocimiento y entendimiento de los ecosistemas terrestres y marinos, no se diga la predicción de sus puntos de quiebre ecosistémico (Tapia-Sisalima, 2018). En este aspecto, la tecnología, identificada como un instrumento fundamental por el concepto de Desarrollo Sostenible representa también otro punto crítico. Aunque resulta indiscutible la necesidad de sustituir el sistema tecnológico usado para el desarrollo económico e industrial del mundo moderno, la elección de tecnologías apropiadas debería resultar de una evaluación de las opciones disponibles al 
momento y del análisis participativo de las necesidades socioeconómicas y ambientales a las que dichas tecnologías deben orientarse (Bermejo, 2014). Sin embargo, estas 'necesidades' están sesgadas, consciente o inconscientemente, hacia un crecimiento económico ilimitado, insostenible. Sumado a ello, no es éticamente adecuado mantener la convicción de que únicamente la tecnología del futuro resolverá los conflictos del presente, pues, aquello implica demasiada incertidumbre, pero, sobre todo, se constituye como una postura social de gran irresponsabilidad.

Adicionalmente, una de las variables determinantes en el Informe Brundtland es el crecimiento demográfico, indicando que no solamente se trata del número de personas sino de hacer que los recursos sean suficientes, sugiriendo que se requieren medidas para limitar las tasas extremas de natalidad. Hace mención, así, a la importancia de proporcionar instrucción con el objetivo de que la población pueda elegir el tamaño de sus familias, manifestando que es una manera de asegurar el derecho humano básico de la libre determinación, en especial, para las mujeres (Ávila, Cadena, \& Almeida, 2012; Naciones Unidas, 1987). Si bien, el enfoque de género es destacable, la insostenibilidad del concepto se enmarca en el sentido de que, además del incremento poblacional, uno de los principales problemas socioeconómicos del Antropoceno radica en la desigual distribución de la riqueza de la sociedad en la que vivimos, pues, podría ser que la cantidad de recursos sea suficiente, pero estos recursos estarían desigualmente distribuidos, viéndose los efectos negativos potenciados por los altos niveles de consumo y desperdicio de las sociedades de los países más industrializados (Jeanrenaud \& Jeanrenaud, 2018; Sánchez, Luque, \& Meira, 2018).

Sin embargo, y, a pesar de sus críticas, el concepto de Desarrollo Sostenible y su teoría han tenido gran aceptación entre organismos internacionales, gobiernos, industria y sociedad civil (Bermejo, 2014), con aquellos interesados por la pobreza, enfatizando la palabra 'desarrollo'; mientras que, aquellos identificados como ambientalistas, exaltando la palabra 'sustentable' (Díaz \& Escárcega, 2009). Su alcance ha sido tal que durante la Asamblea General de la ONU del año 2015 se planteó la Agenda 2030 para el Desarrollo Sostenible, presentada como un plan de acción en favor de las personas, el planeta y la prosperidad (Naciones Unidas, 2015). Desde entonces, la Agenda 2030 ha involucrado a toda la comunidad mundial, convirtiéndose en el punto de referencia y paradigma principal para el desarrollo global futuro. 


\section{La Agenda 2030 para el Desarrollo Sostenible y sus Objetivos}

Es preciso introducir de manera sucinta a la Agenda 2030 y sus Objetivos. En relación a ello, la Asamblea General de la ONU, un foro de deliberación multilateral sobre cuestiones de interés mundial que cumple un rol fundamental en los procesos de adopción de políticas y codificación del derecho internacionales, se caracteriza porque cada uno de sus 193 Estados Miembros está representado por un voto. Resoluciones adoptadas por la Asamblea General, con objetivos concretos propuestos para alcanzar el desarrollo, erradicar la pobreza, proteger el entorno común, etc., han beneficiado a millones de personas alrededor del planeta (Naciones Unidas, 2020). No obstante, si bien, en las últimas décadas los progresos en el ámbito del desarrollo humano han sido extraordinarios, sus beneficios no han sido universales y no todas las vidas han mejorado (Programa de las Naciones Unidas para el Desarrollo, 2016). Al respecto, en el año 2012, durante la Conferencia sobre el Desarrollo Sostenible, los Estados Miembros reconocieron el éxito de los Objetivos de Desarrollo del Milenio (ODM) adoptados en el año 2000, cuyas metas, en el ámbito de abordar ciertas prioridades del desarrollo, se fijaron para el año 2015.

Con este antecedente, los líderes mundiales destacaron la importancia y utilidad de poner en marcha medidas concretas y coherentes en el ámbito del Desarrollo Sostenible, acordando la transición desde los ODM hacia el establecimiento de unos objetivos que aborden e incorporen equilibradamente las tres dimensiones del concepto de sostenibilidad y sus interrelaciones, pero, que sean además concisos, limitados, ambiciosos, globales y universalmente aplicables (Naciones Unidas, 2012; Sachs, 2015). Como resultado, la resolución A/RES/70/1 denominada Transformar nuestro mundo: la Agenda 2030 para el Desarrollo Sostenible, fue presentada por la Asamblea General con el objetivo de asegurar que nadie se quede atrás, cimentándose en cinco enfoques: personas, planeta, prosperidad, paz y alianzas; así como en 17 Objetivos de Desarrollo Sostenible (ODS), de carácter integrado e 
indivisible (Naciones Unidas, 2015). A diferencia de los ODM, los cuales se enfocaron en los países pobres, reconociendo a los países ricos, principalmente, como donadores, los ODS se presentaron como de aplicación universal, con el objetivo de abordar, de mejor manera, el impulso de la acción colectiva frente a la resolución de conflictos comunes en el camino hacia la sostenibilidad. El enfoque holístico del concepto de Desarrollo Sostenible es abordado mediante respuestas sistémicas e integradas, los cuales, sintetizan los ejes fundamentales de las problemáticas identificadas en las diversas conferencias de la ONU a lo largo de décadas (Gómez Gil, 2018).

Los ODS tienen una arquitectura estructurada por 17 objetivos genéricos, materializados en 169 metas medibles a través de 232 indicadores verificables a través de datos estadísticos (Naciones Unidas, 2017). Cada meta vinculada a un ODS se constituye como un objetivo específico mensurable que contribuirá a la consecución de uno o más de los objetivos. Los ODS se destacan por su amplitud y complejidad, pero, también, por su sofisticación. Además de integrar las tres dimensiones del Desarrollo Sostenible y priorizar la lucha contra la pobreza, presentan sólidos vínculos con la defensa de los derechos humanos, con la igualdad de género y el empoderamiento de las mujeres y niñas, con la reducción de las desigualdades y con la eliminación de patrones insostenibles de consumo (Gómez Gil, 2018); Gracias a ellos, desde el 2015 hasta la actualidad, se han logrado avances en algunas áreas; evidencias y datos muestran que aún existen algunas otras que demandan de atención urgente y más efectiva, si se espera alcanzar íntegramente los compromisos propuestos en el ámbito de la sostenibilidad (Naciones Unidas, 2019; OMS/UNICEF, 2017).

Como ya se mencionó, las críticas al concepto de Desarrollo Sostenible, como nuevo paradigma de desarrollo, son variadas. Una de las más reconocidas, es la gran dificultad que implica el hacer operativo al concepto, es decir, reconocer su utilidad práctica como guía o referencia en los procesos de toma de decisiones a cualquier nivel (Torres, Díaz, \& Martínez, 2011). En este mismo sentido, desde la publicación de los ODS, algunos elementos críticos para su correcta implementación han sido identificados, entre los que se incluye: i) la aclaración de los objetivos y sus metas; ii) el mejoramiento del conocimiento y la información técnica disponible; iii) la generación de conocimiento específico en tor- 
no a ellos y su aplicación; iv) el fomento de compromisos y la delimitación de responsabilidades precisas por parte de los Gobiernos; y, v) la solución de problemas por falta de datos (Gómez Gil, 2018), pues, para lograr avances significativos y reales, se necesitan medidas eficaces y eficientes. Otro nivel de críticas radica en que la Agenda 2030 mezclaría objetivos y metas concretas y específicas, con elementos ambiguos e idealistas, además de ofrecer indicaciones vagas respecto a su implementación, manteniendo una mala fama de acuerdos utópicos, sin responsabilidades precisas, que facilitan su incumplimiento (Gómez Gil, 2018).

\section{El caso de la GIRH}

Al considerar la problemática expuesta, con el objetivo de discutir de una manera descriptiva la forma de viabilizar la operatividad del concepto de Desarrollo Sostenible, se hace referencia a su interconexión con el sector hídrico. Esto responde al indisociable vínculo existente entre el agua y las tres dimensiones de la sostenibilidad, al considerar a dicho recurso natural como su núcleo y como la red que interconecta a los ODS propuestos por la Agenda 2030 (Ait-Kadi, 2016; Foster \& Ait-Kadi, 2012). Tanto el Desarrollo Sostenible como la gestión integrada de los recursos hídricos (GIRH), son conceptos complejos, complicados de explicar y fáciles de criticar, que han surgido como respuesta a los crecientes alcances y escalas de los problemas mundiales, brindando marcos de referencia para enfrentar conflictos interconectados (Grigg, 2019).

La relación entre el Desarrollo Sostenible y el sector hídrico se cimienta en el hecho de que tanto el suministro de agua segura como la eliminación de caudales residuales y el acceso a servicios de saneamiento, son elementos básicos en la creación de un ambiente propicio para la mejora de la calidad de vida de las personas, siendo además de fundamentos esenciales, impulsores poderosos del desarrollo humano, los cuales, contribuyen de manera equitativa y equilibrada en las necesidades de una sociedad (Programa Mundial de las Naciones Unidas de Evaluación de los Recursos Hídricos \& ONU-Agua, 2015). 
En respuesta, la Agenda 2030 acordó el ODS 6 que busca "Garantizar la disponibilidad y la gestión sostenible del agua y el saneamiento para todos", reconociendo al agua como fundamental en todos los demás aspectos del Desarrollo Sostenible y a la GIRH como esencial en el progreso y alcance de todos los ODS, entre ellos, los relacionados con la educación, la igualdad de género, la nutrición y la salud (Ait-Kadi, 2016; Naciones Unidas, 2015; OMS/UNICEF, 2017). Es imperativo, entonces, plantear a la GIRH en la posición central e integral de la planificación y el desarrollo social y económico que le pertenece, aceptando y acogiendo sus complejas interconexiones y reconociendo, también, que dicha gestión no se relaciona únicamente con el sector hídrico, pues, en relación a los servicios y beneficios que provee el agua, las tres dimensiones del Desarrollo Sostenible están limitadas por su gestión, finitud y vulnerabilidad (Ait-Kadi, 2016; Programa Mundial de las Naciones Unidas de Evaluación de los Recursos Hídricos \& ONU-Agua, 2015).

La GIRH es definida como un proceso que promueve el desarrollo y la gestión coordinados del agua, la tierra y los recursos relacionados, con el objetivo de maximizar el bienestar económico y social resultante de manera equitativa, sin comprometer la sostenibilidad de los ecosistemas vitales (Smith \& Clausen, 2018). El término 'sostenibilidad' es parte integrante del concepto de la $\mathrm{GIRH}$, en tanto que los conflictos que amenazan la demanda mundial de agua, que incrementan el estrés hídrico y que fomentan desafíos intersectoriales en las sociedades humanas en relación al agua, son los mismos que fundamentan el paradigma del Desarrollo Sostenible (De Albuquerque, 2013; Van Leeuwen, Hofman, Driessen, \& Frijns, 2019). En este mismo sentido, sus críticas son similares, en relación a que la GIRH, también, se vería limitada respecto a la entrega de resultados concretos, así como a una falta de operatividad que le permita erigirse como una guía para los procesos de toma de decisiones. Empero, la adopción de los ODS y el reconocimiento del potencial de la GIRH para la movilización de sinergias entre los objetivos y las metas, han incrementado la presión para convertirla en un proceso más tangible (Smith \& Clausen, 2018).

En este contexto, la acción práctica, la resolución pragmática de problemas y la descentralización en la toma de decisiones, podrían ser el camino para hacer operacional tanto a la GIRH como al concepto de Desarrollo Sostenible (Smith \& Clausen, 2018). En relación a ello, 
los indicadores de gestión se constituyen como una fuerte alternativa para la integración conceptual y para pasar de términos teóricos a acciones operacionales (Torres et al., 2011). Los conceptos de Desarrollo Sostenible y de GIRH, así como su funcionamiento, pueden parecer muchas veces vagos y abstractos, y, al reconocer que la evaluación del desempeño de todo proceso es parte fundamental de su planificación y control, la falta de medidas estándar para el seguimiento del éxito de sus planes y proyectos se constituye como uno de sus principales problemas (AWRA, 2011; Buccheri \& Comellas, 2011). En respuesta, desde diferentes ámbitos se ha propuesto el análisis a través de indicadores, los cuales, permiten la cuantificación y simplificación de un fenómeno complejo al representar de manera concreta sus cualidades en un momento determinado (Buccheri \& Comellas, 2011; INBO \& GWP, 2012; Van Leeuwen et al., 2019). Los indicadores están pensados tanto en profesionales como en responsables políticos y público general como objetivo y se constituyen como un paso clave en el proceso de recopilación de información que facilita la planificación y la gestión, tanto del agua como de otros recursos. En el ámbito de la sostenibilidad, la operatividad de la GIRH debe conciliar sus principios fundamentales con la acción práctica, a través de soluciones que provengan desde todos los sectores involucrados, facilitando el aprendizaje social respaldado por datos, la comunicación, el empoderamiento y el aprendizaje práctico. Además, debe considerar mecanismos operativos para acercar el planteamiento de estrategias con la resolución de problemas, a través de plataformas que reúnan a las partes interesadas para la toma de decisiones de manera colaborativa (Smith \& Clausen, 2018; Wang, Davies, \& Liu, 2019). Todo esto puede fundamentarse en los análisis a través de indicadores de gestión, lo que conduciría a una mejor planificación, ofreciendo información valiosa y práctica que respalda la toma de decisiones para el Desarrollo Sostenible.

En relación a ello, si bien los ODS propuestos en la Agenda 2030 tienen una compleja arquitectura estructurada por 17 objetivos genéricos, estos se materializan en 169 metas medibles a través de 232 indicadores (Naciones Unidas, 2017). Ya que el Desarrollo Sostenible se constituye como una cuestión de justicia social, en la cual, en relación a la equidad intrageneracional e intergeneracional que persigue, los beneficios de los progresos alcanzados en materia de desarrollo humano deben ser para todas las personas considerando, de esta manera, una equidad 
distributiva, es preciso que su seguimiento se fundamente en datos desglosados que faciliten el alcance de aquello propuesto por la Agenda 2030. Empero, en búsqueda de la operatividad, tal como el caso de la GIRH, la escala de los indicadores de los ODS podría reducirse hacia características más locales, considerando a todas las partes involucradas, por pequeña que sea su presencia, en los procesos de toma de decisiones. Los enfoques holísticos y complejos son comunes, pero, así también, lo podrían ser sus dinámicas y abordajes hacia la operatividad. Es importante, finalmente, que los análisis a través de indicadores no pierdan de vista las tres dimensiones en las que se fundamenta el concepto de Desarrollo Sostenible.

\section{Consideraciones Finales}

En el contexto del Antropoceno y su inherente y complejo problema economía - desarrollo - ambiente, la imprescindible búsqueda de soluciones a las crisis planetarias ha dirigido la agenda global, desde hace casi medio siglo, por el camino del Desarrollo Sostenible como paradigma para el desarrollo a largo plazo. Este concepto se fundamenta en tres pilares: desarrollo económico, desarrollo social y protección medioambiental; sin embargo, al igual que la teoría capitalista, persigue un crecimiento económico ilimitado, considerando a la Naturaleza como el medio para lograrlo. En tal razón y debido a las restricciones generadas desde intereses económicos y políticos, muchas veces el concepto ha sido tergiversado, únicamente, hacia un discurso políticamente correcto.

Gran parte de la población mundial ha sido socializada, educada y madurada en mundos modernos o bajo su influencia y dominio, por lo que comúnmente se concibe al desarrollo capitalista como el único camino posible para la sociedad humana. Superar este pre-condicionamiento se convierte en una tarea complicada, pues, nuevas alternativas pueden resultar inconcebibles e, incluso, inconmensurables con la nuestra. En relación a ello, y, a pesar de sus críticas, el concepto de Desarrollo Sostenible y su teoría han tenido gran aceptación a nivel general, con un alcance y penetración tal, que la actual agenda de desarrollo 
de la ONU se fundamenta en él como punto de referencia principal. La Agenda 2030 para el Desarrollo Sostenible se ha presentado con el objetivo de asegurar que nadie se quede atrás y se fundamenta en una arquitectura estructurada por 17 objetivos genéricos, materializados en 169 metas medibles a través de 232 indicadores verificables a través de datos estadísticos, que, si bien han potenciado avances en algunas áreas, evidencias y datos muestran que aún existen algunas otras que demandan de atención urgente y más efectiva, si se espera alcanzar íntegramente los compromisos propuestos en el ámbito de la sostenibilidad.

Al tener en cuenta las principales críticas del Desarrollo Sostenible, entre ellas, su dificultad de hacerlo operativo, así como un funcionamiento que puede parecer abstracto, con el objetivo de discutir de una manera descriptiva la forma de viabilizar este concepto, se hizo referencia a su interconexión con el sector hídrico, el cual, ha sido reconocido como su núcleo y como la red que conecta los ODS. En relación a ello, el proceso de la GIRH también es considerado como complicado de explicar y fácil de criticar, pero, al igual que el Desarrollo Sostenible, ha surgido como respuesta a los alcances y escalas de los conflictos mundiales, proveyendo marcos de referencia para enfrentarlos, que, no obstante, las condiciones exigen que sean más tangibles y operativos.

En el contexto planteado, el análisis a través de indicadores de gestión parecería ofrecer una respuesta adecuada que facilita el aprendizaje social, la comunicación, el empoderamiento, la inclusión de todas las partes interesadas y la consideración de las condiciones locales; y, conduciría a una mejor planificación, al permitir el conocimiento del estado actual de problemas complejos, ofreciendo, además, información valiosa y práctica en la toma de decisiones. Enfoques holísticos y complejos son comunes entre Desarrollo Sostenible y GIRH, por lo que sus dinámicas y abordajes hacia la operatividad en el ámbito de la sostenibilidad también podrían serlo. 


\section{Referencias bibliográficas}

Acosta \& Martínez (Eds.), La Naturaleza con Derechos. De la filosofía a la política. (pp. 239258). Quito: AbyaYala y Universidad Politécnica Salesiana.

Ait-Kadi, M. (2016). Water for development and development for water: realizing the Sustainable Development Goals (SDGs) vision. Aquatic Procedia, 6, 106-110. https://doi. org/10.1016/j.aqpro.2016.06.013

Alburquerque, F. (2010). Economía Ecológica y Desarrollo Económico Local.

Alburquerque, F. (2013). Economía del desarrollo y desarrollo territorial.

Ávila, A., Cadena, M., \& Almeida, M. (2012). Guía de derechos económicos, sociales, culturales y ambientales. (M. J. Avilés, Ed.). Quito: Escuela de Formación Político Feminista.

AWRA (2011). Integrated Water Resources Management in the US. In AWRA Board of Directors.

Bermejo, R. (2014). Del desarrollo sostenible según Brundtland a la sostenibilidad como biomimesis. Bilbao: Instituto de Estudios sobre Desarrollo y Cooperación Internacional.

Buccheri, M., \& Comellas, E. (2011). Indicadores para el monitoreo y evaluación hacia la GIRH, 25.

Carpio, P. (2019). Economía Política del Cambio Climático y Desarrollo - Maestría en Estudios Socioambientales, Universidad del Azuay. Cuenca.

De Albuquerque, C. Informe de la Relatora Especial sobre el derecho humano al agua potable y el saneamiento (2013). https://undocs.org/A/HRC/24/44

Díaz, R., \& Escárcega, S. (2009). Desarrollo Sustentable. Una oportunidad para la vida (Primera Ed). México: McGraw-Hill.

Foster, S., \& Ait-Kadi, M. (2012). Integrated Water Resources Management (IWRM): How does groundwater fit in? Hydrogeology Journal, 20(3), 415-418. https://doi.org/10.1007/ s10040-012-0831-9

Gómez Gil, C. (2018). Objetivos de Desarrollo Sostenible (ODS): una revisión crítica. Papeles de Relaciones Ecosociales y Cambio Global, (140), 107-118.

Grigg, N. (2019). IWRM and the Nexus Approach: Versatile Concepts for Water Resources Education. Journal of Contemporary Water Research \& Education, (166), 24-34. https:// doi.org/10.1111/j.1936-704X.2019.03299.x 
Gudynas, E. (2011). Los derechos de la Naturaleza en serio. Respuestas y aportes desde la ecología política. In A.

INBO \& GWP (2012). The handbook for Integrated Water Resources Management in transboundary basins of rivers, lakes and aquifers. París: International Network of Basin Organizations - INBO.

Jeanrenaud, S., \& Jeanrenaud, J. (2018). Navigating the Anthropocene: Insights from the Wisdom of the Corpus Hermeticum. In M.-A. Crumplin (Ed.), Deep Philosophy, Deep Ecology (pp. 117-145). Prometheus Trust.

Méndez, J. (2005). Doctrinas Económicas. In Fundamentos de Economía (IV, pp. 59-86). México: McGraw-Hill Interamericana, S.A.

Molina, M., Sarukhán, J., \& Carabias, J. (2017). El cambio climático: causas, efectos y soluciones. Ciudad de México: Fondo de Cultura Económica.

Naciones Unidas. Proceso de elaboración de la perspectiva ambiental hasta el año 2000 y más adelante (1982). A/RES/38/161.

Naciones Unidas. (1987). Informe de la Comisión Mundial sobre el Medio Ambiente y el Desarrollo. https://undocs.org/es/A/42/427

Naciones Unidas. (2012). El futuro que queremos. Conferencia de las Naciones Unidas sobre el Desarrollo Sostenible (p. 59). Rio de Janeiro. Retrieved from https://rio20.un.org/sites/ rio20.un.org/files/a-conf.216-I-1_spanish.pdf.pdf

Naciones Unidas. Transformar nuestro mundo: la Agenda 2030 para el Desarrollo Sostenible (2015). A/RES/70/1. https://www.un.org/ga/search/view_doc.asp?symbol=A/ $\mathrm{RES} / 70 / 1 \&$ Lang $=\mathrm{S}$

Naciones Unidas. Labor de la Comisión de Estadística en relación con la Agenda 2030 para el Desarrollo Sostenible. Anexo: Marco de indicadores mundiales para los Objetivos de Desarrollo Sostenible y metas de la Agenda 2030 para el Desarrollo Sostenible (2017). A/RES/71/313. https://undocs.org/es/A/RES/71/313

Naciones Unidas. (2019). Progresos realizados para lograr los Objetivos de Desarrollo Sostenible: Informe del Secretario General. https://undocs.org/es/E/2019/68

Naciones Unidas. (2020). Asamblea General de las Naciones Unidas. Retrieved February 18, 2020, from https://www.un.org/es/ga/ 
OMS/UNICEF. (2017). Progresos en materia de agua potable, saneamiento e higiene: informe de actualización de 2017 y línea de base de los ODS. (A. Grojec, Ed.). Ginebra: Organización Mundial de la Salud y Fondo de las Naciones Unidas para la Infancia. washdata. org/sites/default/files/documents/reports/2018-01/JMP-2017-report-final.pdf

PNUD (2016). Informe sobre Desarrollo Humano 2016. Desarrollo humano para todas las personas. Programa de las Naciones Unidas para el Desarrollo.

PNUD (2015). Programa Mundial de las Naciones Unidas de Evaluación de los Recursos Hídricos, \& ONU-Agua The United Nations world water development report 2015: water for a sustainable world. UNESCO. http://www.unwater.org/publications

Rodríguez, V., \& Sánchez, R. (2019). Reflexiones críticas de la sostenibilidad como construcción políticamente correcta del desarrollo. Pluriversidad, (4), 133-150. https://doi. org/10.31381/pluriversidad.v4i4.2775

Sachs, J. (2015). The age of sustainable development. Columbia University Press.

Salas, R. (2018). Un análisis crítico al marco conceptual del desarrollo sostenible y sus herramientas de medición. En-Contexto 6 (8), 171-184.

Sánchez, E., Luque, D., \& Meira, P. (2018). ¿Es la Educación para el Desarrollo Sostenible (EDS) la respuesta a la crisis socio-ambiental?: una reflexión desde la sospecha. XXXVII Seminario Interuniversitario de Teoría de la Educación: Educación en la Sociedad de Conocimiento y el Desarrollo Sostenible (pp. 325-362). Universidad de La Laguna.

Smith, M., \& Clausen, T. J. (2018). Revitalising IWRM for the 2030 Agenda. In World Water Council Challenge Paper for the High-Level Panel on IWRM at the 8th World Water Forum.

Tapia-Sisalima, J. (2018). El páramo del Cajas: ecosistema esencial para el abastecimiento de agua al cantón Cuenca. Maestría en Estudios Socioambientales. Módulo: Adaptación, Mitigación y Vulnerabilidad en Ecosistemas Andinos y Amazónicos - Universidad del Azuay. Cuenca.

Torres, P., Díaz, B., \& Martínez, D. (2011). Los indicadores, una herramienta en la gestión integrada de los recursos hídricos en México. JAINA Boletín Informativo, 22(1), 31-40.

Van Leeuwen, K., Hofman, J., Driessen, P., \& Frijns, J. (2019). The Challenges of Water Management and Governance in Cities. Water, 11(6), 1180-1185. https://doi. org/10.3390/w11061180 
Wang, K., Davies, E. G. R., \& Liu, J. (2019). Integrated water resources management and modeling: A case study of Bow river basin, Canada. Journal of Cleaner Production, 240, 118242. https://doi.org/10.1016/j.jclepro.2019.118242 